\title{
U.S. vaccine and immune globulin product shortages, 2001-15
}

Victoria C. Ziesenitz, M.D., M.Sc., Department of Pediatric Pharmacology and Pharmacometrics, University of Basel Children's Hospital, Basel, Switzerland, and Department of Pediatric Cardiology, University Children's Hospital, Heidelberg, Germany.

Maryann Mazer-Amirshahi, Pharm.D., M.D., M.P.H., Department of Emergency Medicine, MedStar Washington Hospital Center, Washington, DC, and Georgetown University School of Medicine, Washington, DC

Mark S. Zocchi, M.P.H., Center for Healthcare Innovation and Policy Research, George Washington University, Washington, DC

Erin R. Fox, Pharm.D., Drug Information Service, University of Utah Health Care, Salt Lake City, UT, and College of Pharmacy, University of Utah, Salt Lake City, UT.

Larissa S. May, M.D., M.S., M.S.P.H., Department of Emergency Medicine, University of California Davis, Sacramento, CA
Address correspondence to Dr. Ziesenitz (ziesenitz.md@gmail.com).

Copyright (C) 2017, American Society of Health-System Pharmacists, Inc. All rights reserved. 1079-2082/17/1102-1879.

DOI 10.2146/ajhp170066

Purpose. Trends in shortages of vaccines and immune globulin products from 2001 through 2015 in the United States are described.

Methods. Drug shortage data from January 2001 through December 2015 were obtained from the University of Utah Drug Information Service. Shortage data for vaccines and immune globulins were analyzed, focusing on the type of product, reason for shortage, shortage duration, shortages requiring vaccine deferral, and whether the drug was a single-source product. Inclusion of the product into the pediatric vaccination schedule was also noted.

Results. Of the 2,080 reported drug shortages, 59 (2.8\%) were for vaccines and immune globulin products. Of those, 2 shortages (3\%) remained active at the end of the study period. The median shortage duration was 16.8 months. The most common products on shortage were viral vaccines (58\%), especially hepatitis $A$, hepatitis $B$, rabies, and varicella vaccines (4 shortages each). A vaccine deferral was required for 21 shortages $(36 \%)$, and single-source products were on shortage 30 times $(51 \%)$. The most common reason for shortage was manufacturing problems $(51 \%)$, followed by supply-and-demand issues (7\%). Thirty shortages $(51 \%)$ were for products on the pediatric schedule, with a median duration of 21.7 months.

Conclusion. Drug shortages of vaccines and immune globulin products accounted for only $2.8 \%$ of reported drug shortages within a 15year period, but about half of these shortages involved products on the pediatric vaccination schedule, which may have significant public health implications.

Keywords: bacterial vaccines, immunization, immunoglobulins, supply and distribution, vaccines, viral vaccines

Am J Health-Syst Pharm. 2017; 74:1879-86

P rescription drug shortages, defined by the Food and Drug Administration (FDA) as "a period of time when the demand or projected demand for the drug within the United States exceeds the supply of the drug," have become increasingly prevalent over the past 2 decades. ${ }^{1,2}$ Manufacturing and quality-control problems, as well as economic factors, have resulted in frequent supply disruptions. ${ }^{2}$ Prescription drug shortages have affected multiple specialties, including oncology, emergency medicine, and infec- tious diseases..$^{3-5}$ Data have also revealed that sterile injectable products are more commonly in short supply compared with products for oral or topical administration..$^{2-5}$

Millions of vaccinations are administered each year for infection prevention and control. ${ }^{6}$ Vaccine shortages can have significant public health and clinical implications, as they may result in individuals not being fully vaccinated according to the recommended schedule, thereby compromising herd immunity and 
creating the potential for epidemic outbreaks of communicable diseases. Vaccine shortages may affect not only the health of adult patients seeking secondary vaccinations but that of vulnerable infants and children who are in need of primary vaccinations, with a potential significant effect on communicable disease morbidity and mortality due to reduced herd immunity.

In the case of a vaccine shortage, the Centers for Disease Control and Prevention (CDC) may recommend to postpone or omit a dose of a recommended booster vaccination in order to allow unvaccinated children to receive a first dose of the vaccine in short supply. ${ }^{6}$ Data describing how drug shortages have affected vaccine and immune globulin availability are limited. Previous reports have focused on the availability of Haemophilus influenzae type $\mathrm{b}$ (Hib), pneumococcal, and influenza vaccines. ${ }^{7-11}$ We assessed the shortages of vaccines and immune globulins in the United States from 2001 through 2015.

\section{Methods}

The University of Utah Drug Information Service (UUDIS) has been collecting national drug shortage data since January 2001 and publishes critical drug shortage information on a public website (www.ashp.org/shortage) hosted by the American Society of Health-System Pharmacists (ASHP). UUDIS defines a shortage as "a supply issue that affects how the pharmacy prepares or dispenses a drug product or influences patient care when prescribers must use an alternative agent". ${ }^{12}$ This definition differs slightly from, but is more inclusive than, FDA's definition of a shortage. For example, a shortage of prefilled syringes of a specific product may cause significant logistic and safety issues, even if the same medication is available in vials.

The Government Accountability Office (GAO) considers UUDIS data to be the most comprehensive and reliable source of drug shortage information and has used UUDIS data for 4 re-

\section{KEY POINTS}

- From 2001 through 2015, half of the vaccine shortages affected the pediatric vaccine schedule.

- Nearly $90 \%$ of vaccines had multiple shortages during the study period.

- The mitigation of vaccine shortages is crucial for public health, especially shortages of products on the pediatric vaccination schedule.

ports published on drug shortages. ${ }^{2,13}$ Detailed methods describing UUDIS have been previously published. ${ }^{14}$ Briefly, UUDIS receives voluntary reports of drug and vaccine shortages via the reporting feature on the ASHP website. Clinical pharmacists at UUDIS research each shortage reported to verify that the shortage actually exists. This research includes determining all potential manufacturers of a reported drug or vaccine in shortage and all drug presentation National Drug Codes (NDCs). Next, each manufacturer is contacted to determine which NDCs are in shortage at the national level. The manufacturers are also asked for a reason for the shortage as well as an estimated release date. If most manufacturers are having a national shortage, then UUDIS will post information on the ASHP drug shortage website noting which products are affected, which products are available, specific methods for accessing the product, reasons for the shortage, estimated resupply dates, and, if applicable, implications for patient care, safety concerns, alternatives, and management strategies. UUDIS considers a shortage to be resolved when all suppliers have all presentations available or have discontinued their products. UUDIS also follows FDA's drug shortage website and will generally list shortages as re- solved when FDA considers the shortages resolved.

UUDIS collects the following drug shortage data using Excel (Microsoft Corp., Redmond, WA): generic product name, therapeutic category, date shortage began (date UUDIS was notified), date shortage was resolved, duration of shortage, reason for the shortage, controlled substance schedule (if applicable), and whether the drug is an injectable product. Drug shortages that occur due to a product discontinuation or withdrawal from the market have the same start and stop dates for a duration of 0 days.

The data set was restricted to shortages that occurred between January 1, 2001, and December 31, 2015, and was analyzed in 2016. Members of the study team identified shortages that affected vaccines and immune globulins. All discrepancies were discussed until a consensus was reached. Shortage data were analyzed, focusing on the type of product, reason for the shortage, shortage duration, shortages requiring vaccine deferral, and whether the drug was a single-source product (produced by 1 manufacturer). Inclusion of the product into the pediatric vaccination schedule was also noted. The availability of a substitute therapy and whether the alternative was also affected by a shortage at any time during the study period, as well as multiple shortages (those that were resolved but then another shortage occurred) of the same vaccine were also noted. For some portions of the analysis, products were grouped together based on vaccine category, such as bacterial vaccines, viral vaccines, combination products, immune globulins, or toxoids. Shortage length was analyzed using Hodges-Lehmann median differences and the KruskalWallis test for comparisons of more than 2 groups. Negative binomial regression was used to determine if the trend in new shortages observed each year was significant over the study period. Discontinued products were not included in analyses of shortage duration $(n=3)$. Data were analyzed using 
Stata, version 14.0 (Stat Corp., College Station, TX). These data do not meet the definition of human subjects' research and therefore were exempt from institutional review board review.

\section{Results}

Number and duration of shortages. A total of 2,080 drug shortages were reported from January 2001 through December 2015, 59 (2.8\%) of which involved vaccines or immune globulins. The number of products on shortage decreased from 11 in 2001 to 2 in $2015(p=0.08)$ (Figure 1). The median shortage duration was 16.8 months (interquartile range [IQR], 5.8-27.3 months). The median number of new shortages reported annually was 3 (IQR, 2-5). By the end of the study period, 2 products remained on active shortage (meningococcal vaccine for 230 days; yellow fever vaccine for 119 days). The median duration for the 57 resolved shortages was 18.3 months (IQR, 6.1-27.4 months). Fiftythree vaccines or immune globulins (90\%) were the subject of multiple shortages during the study period. Rabies immune globulin had the longest shortage duration for any vaccine or immune globulin (2,717 days, from August 16, 2007, to January 23, 2015).

Thirty shortages (51\%) involved vaccines on the pediatric schedule, with a median shortage duration of 21.7 months (IQR, 8.0-28.1 months). A vaccine deferral was required for 21 shortages (36\%), and single-source products were on shortage 30 times. Single-source products were involved in $51 \%$ of shortages, with a median shortage duration of 19.8 months (IQR, 11.1-26.7 months). Shortage durations did not differ significantly among the product types examined.

Types of products involved in shortages. Of the 59 vaccine shortages during the study period, 34 (58\%) involved viral vaccines, 18 (31\%) involved bacterial vaccines, and 7 (12\%) involved toxoids. Combination products accounted for 9 (15\%) of reported shortages. Eight (14\%) products affected by shortages were immune globulins (Table 1). Of all shortages, combination products had the longest median shortage duration (25.7 months; IQR, 16.3-36.8 months); however, this duration was not significantly different than that for other types of products $(p=0.202)$. The most common products with multiple shortages were viral vaccines, particularly hepatitis A, hepatitis B, rabies, and varicella vaccines, with 4 shortages each (Table 2).

Reasons for shortages. The most commonly reported reason for a shortage was manufacturing problems (51\%), followed by supply-anddemand issues (7\%) (Table 3). A reason was not provided for 18 cases $(31 \%)$ of vaccine shortages. Shortage duration differed significantly depending on the reason given for the shortage ( $p=0.004$, Kruskal-Wallis test). The longest durations were for products on shortage due to manufacturing problems (median, 22.8 months; IQR, 16.8-31.1 months).

\section{Discussion}

Although the number of vaccine and immune globulin shortages (59 [2.9\%]) was low compared with the total number of drug shortages $(2,080)$ during the study period, shortages of these products can have far-reaching implications. Thirty (51\%) of these shortages affected the pediatric vaccine schedule, and it is unknown how many children did not receive their full vaccination series due to these shortages. The National Immunization Survey reported that the vaccination coverage for diphtheria, tetanus, and acellular pertussis (DTaP) or measles, mumps, and rubella (MMR) was not affected by these shortages at a national level. ${ }^{15,16}$ It is unknown whether vaccination rates for vaccines not on shortage remained stable, as any decrease in these rates would imply that the shortages had a causal role.

Nearly $90 \%$ of vaccines had multiple shortages during the study period, including important childhood vaccines such as varicella and hepatitis B (Table 2). Recurrent shortages of the same products suggest systematic errors in the manufacturing process. Vaccines and immune globulin products have more complex manufacturing requirements than do simple molecules and oral products. Problems with manufacturing these products

Figure 1. Total number of vaccine and immune globulin products in short supply from 2001 through 2015.

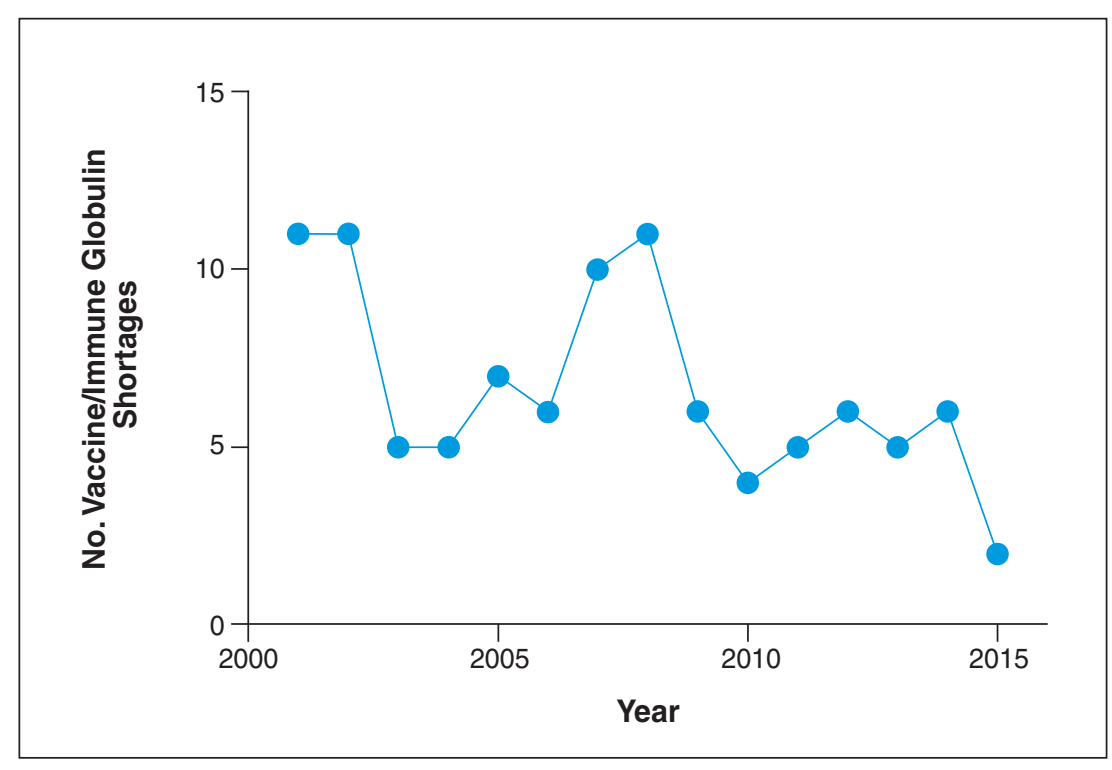


Table 1. Types of Vaccine Products Affected by Drug Shortages ${ }^{a}$

\begin{tabular}{lccc}
\hline \multicolumn{1}{c}{ Product Type $^{\text {b }}$} & $\begin{array}{c}\text { No. (\%) } \\
\text { Shortages }\end{array}$ & $\begin{array}{c}\text { Median Shortage } \\
\text { Duration in Months (IQR) }\end{array}$ & $\begin{array}{c}\text { Median (95\% Cl) } \\
\text { Difference vs. Other } \\
\text { Product Types, in Months }\end{array}$ \\
\hline All products & $59(100)$ & $16.8(5.8-27.3)$ & $\ldots$ \\
\hline Bacterial vaccines & $18(31)$ & $22.6(11.1-27.4)$ & $5.7(-2.5$ to 16.2) \\
\hline Combination products & $9(15)$ & $25.7(16.3-36.8)$ & $9.0(-3.9$ to 20.2) \\
\hline Immune globulins & $8(14)$ & $5.1(3.7-13.9)$ & $-6.8(-17.6$ to 0.0) \\
\hline Toxoids & $7(12)$ & $23.3(4.1-36.8)$ & $2.2(-18.2$ to 21.5) \\
\hline Viral vaccines & $34(58)$ & $16.6(6.1-27.8)$ & $-0.7(-10.5$ to 5.7) \\
\hline Products on pediatric schedule & $30(51)$ & $21.7(8.0-28.1)$ & $5.0(-0.7$ to 14.1) \\
\hline Vaccine with deferral required during shortage & $21(36)$ & $22.3(11.1-27.4)$ & $5.6(-2.2$ to 13.7) \\
\hline Single-source products & $30(51)$ & $19.8(11.1-26.7)$ & $3.5(-3.5$ to 11.9) \\
\hline $\begin{array}{l}\text { alQR }=\text { interquartile range, } \mathrm{Cl}=\text { confidence interval. } \\
\text { bSome products may fit into more than 1 category. }\end{array}$ & & &
\end{tabular}

accounted for $51 \%$ of the reported shortages (Table 3). Shortages of vaccines and immune globulin products usually take longer to resolve, and in many cases the actual manufacturing time of these products can take several months. This means that shortages of these products will be lengthy. The mean duration of shortage was well over 1 year (median duration of resolved shortages, 18.3 months), with the longest shortage durations of over 2 years for combination products (median, 25.7 months), including shortages for vaccines on the pediatric schedule, and almost 2 years for toxoids (median, 23.3 months) (Table 1). The advantage of combination vaccines is that they reduce the number of injections a child needs to in order to be fully immunized against several diseases. If individual vaccines are used due to a shortage of combination vaccines, the number of injections required for a child would increase, which might lead to objections against vaccinations in general.

Single-source products. Products manufactured by a single company or facility are at higher risk of a drug shortage, because any disruption in the manufacturing process typically results in a shortage. ${ }^{17}$ GAO's latest report on drug shortages also notes that products with few suppli- ers are at greater risk of a drug shortage. ${ }^{18}$ In this analysis, $51 \%$ of products on shortage were produced by a single manufacturer, and 5 products $(8 \%)$ were affected by; at least 3 recurrent shortages (varicella, measles, mumps, rubella [MMR]; typhoid). Vaccines and immune globulin products are often single-source products or only supplied by 3 or fewer manufacturers. For example, in 2006, there was only 1 licensed manufacturer in the United States distributing MMR vaccine; varicella vaccine; tetanus and diphtheria vaccine; inactivated polio virus vaccine; meningococcal conjugate vaccine; and pneumococcal conjugate vaccines. ${ }^{19}$

Implications of deferrals. Shortages of vaccines have the potential to cause profound public health implications. The Advisory Committee on Immunization Practices (ACIP) recommends that clinicians evaluate vaccination status at each patient visit and avoid delaying vaccinations (e.g., deferral for mild illness). ${ }^{6}$ Shortages of vaccines can result in deferrals and delays of over a year for vaccinations because many school-aged children only see a provider annually and most vaccine shortages last longer than 1 year. In this analysis, a vaccine deferral was required for $36 \%$ of shortages. In addition, multiple shortages might have a greater impact on children who get their first vaccination series because recommended vaccinations might be deferred more than once.

Shortages of vaccines have also resulted in changes to school vaccination requirements. ${ }^{20} \mathrm{~A}$ publication describing 2 shortages of pneumococcal vaccine (heptavalent pneumococcal conjugate vaccine, PCV7) in 2001 and 2004 analyzed whether the vaccination behavior of pediatricians in Cincinnati was changed through the shortages. Only $66 \%$ of healthy children were documented as having received their first 2 doses of pneumococcal vaccine on time, compared with $74 \%$ of healthy children during nonshortage periods. ${ }^{10}$ An analysis of the vaccination coverage rates of Hib during the 2008-09 vaccine shortage revealed $7.8-10.3 \%$ lower coverage rates compared with periods without a shortage..$^{9}$ Results of another study found a higher Hib carrier rate in Atlanta due to the vaccination deferral during the 2008-09 Hib vaccine shortage. ${ }^{8}$

Unfortunately, our data show that many of the problems highlighted in GAO's 2002 report on vaccine shortages still exist, such as manufacturing problems, demand exceeding supply, and a lack of redundancy in the manufacturing process. ${ }^{20}$ In addition, vac- 
Table 2. Characteristics of Vaccines and Immune Globulins Affected by Shortages, 2001-15

\begin{tabular}{|c|c|c|c|c|}
\hline Vaccine or Immune Globulin & $\begin{array}{l}\text { No. } \\
\text { Shortages }\end{array}$ & $\begin{array}{l}\text { Single } \\
\text { Source? }\end{array}$ & $\begin{array}{c}\text { Shortage } \\
\text { Active on } \\
\text { December 31, } \\
\text { 2015? }\end{array}$ & $\begin{array}{c}\text { Included } \\
\text { on } \\
\text { Pediatric } \\
\text { Schedule? }\end{array}$ \\
\hline Hepatitis A & 4 & No & No & No \\
\hline Hepatitis B & 4 & No & No & Yes \\
\hline Rabies & 4 & No & No & No \\
\hline Varicella & 4 & Yes & No & Yes \\
\hline Haemophilus influenzae type b & 3 & No & No & Yes \\
\hline Measles & 3 & Yes & No & Yes \\
\hline Mumps & 3 & Yes & No & Yes \\
\hline Rabies immune globulin & 3 & No & No & No \\
\hline Rubella & 3 & Yes & No & Yes \\
\hline Typhoid & 3 & Yes & No & No \\
\hline Cytomegalovirus immune globulin & 2 & Yes & No & No \\
\hline Diphtheria, tetanus, and acellular pertussis & 2 & No & No & Yes \\
\hline Hepatitis B immune globulin & 2 & No & No & No \\
\hline Influenza & 2 & No & No & Yes \\
\hline Meningococcal (MCV4) & 3 & Yes & Yes & Yes \\
\hline Measles, mumps, rubella, and varicella & 2 & Yes & No & Yes \\
\hline Pneumococcal (PCV7) & 2 & Yes & No & Yes \\
\hline $\begin{array}{l}\text { Diphtheria and tetanus toxoids and acellular pertussis vaccine } \\
\text { adsorbed }\end{array}$ & 2 & No & No & No \\
\hline Diphtheria, tetanus, pertussis, and poliovirus antigens & 1 & No & No & Yes \\
\hline $\begin{array}{l}\text { Diphtheria, tetanus, pertussis, poliovirus, and H.influenzae type b } \\
\text { antigens }\end{array}$ & 1 & Yes & No & Yes \\
\hline Measles, mumps, and rubella & 1 & Yes & No & Yes \\
\hline Pneumococcal (V23) & 1 & Yes & No & No \\
\hline Yellow fever & 2 & Yes & Yes & No \\
\hline Varicella immune globulin & 1 & No & No & No \\
\hline Tetanus & 1 & Yes & Yes & No \\
\hline
\end{tabular}

cine shortages may have a disparate effect on vulnerable populations. For example, among children receiving vaccinations in public clinics, those living outside of urban areas, children in the South, and American Indian/ Alaska Native children were less likely to have received the fourth dose of the DTaP vaccine during the shortage. ${ }^{22,23}$

Mitigation strategies. There are several potential strategies that may mitigate the impact of vaccine shortages, such as early notification about
Table 3. Reported Reasons for Vaccine and Immune Globulin Shortages ${ }^{a}$

\begin{tabular}{|c|c|c|}
\hline Reason for Shortage & $\begin{array}{c}\text { No. (\%) } \\
\text { Shortages }\end{array}$ & $\begin{array}{l}\text { Median (IQR) Duration } \\
\text { of Shortage, in Months }\end{array}$ \\
\hline Discontinued product & $3(5)$ & NA \\
\hline Manufacturing problem & $30(51)$ & $22.8(16.8-31.1)$ \\
\hline Supply-and-demand issues & $4(7)$ & $16.4(5.8-22.1)$ \\
\hline Otherc & $4(7)$ & $6.1(4.5-15.5)$ \\
\hline Unknown & $18(31)$ & $7.8(5.1-14.8)$ \\
\hline \multicolumn{3}{|c|}{$\begin{array}{l}\text { a }{ }^{a} R=\text { interquartile range, } \mathrm{NA}=\text { not applicable. } \\
{ }^{\mathrm{b}} \chi^{2}=13.199 \text { with } 3 \text { d.f. }(p=0.004) \text {, Kruskal-Wallis test. } \\
{ }^{\mathrm{N} N a t u r a l ~ d i s a s t e r ~}(n=2) \text {, shortage of raw materials }(n=1) \text {, and product recall }(n=1) \text {. }\end{array}$} \\
\hline
\end{tabular}


foreseeable shortages, vaccine deferrals, vaccine stockpiles, and importation of commercial alternatives. ${ }^{24}$

Manufacturers should provide early notification about expected shortages to ensure that this information is included in current recommendations. ${ }^{25}$ Providers should become more vigilant regarding anticipated shortages and proactively incorporate recommended strategies regarding existing supplies and future distribution. Unlike shortages of oncology or emergency drugs, vaccine shortages do not usually require institutional responses. Decisions on vaccine deferrals should only be made on an institutional basis in emergency situations (e.g., by vaccinating only high-risk patients) because vaccines are not usually used during emergencies. Instead, recommendations on deferrals should be issued on a state or national level. In the past, CDC, together with the American Academy of Pediatrics and the American Academy of Family Physicians, issued vaccine deferral recommendations, asking providers to withhold a third or fourth dose of PCV7 during the 2003-04 shortage. ${ }^{21}$

In another study, good adherence of pediatricians to vaccination recommendations was reported, with $91 \%$ of assessed care providers stating that high-risk patients were fully vaccinated. At the same time, pediatricians whose practice experienced no vaccine shortage were more likely to administer a fourth dose of PCV7 vaccine despite recommendations to the contrary. ${ }^{26}$ This highlights the complexities of implementing vaccine deferrals when the vaccine supply is not distributed equally.

In order to keep track of patients who miss a vaccine booster during a shortage, proper surveillance measures should be implemented. During the 2003-04 shortage of PCV7, systems to track children who missed doses due to redistribution of vaccines at the national level were reported in approximately two thirds of the practices that deferred a vaccination..$^{21,26}$
Influenza vaccine shortages were reported for patients at high risk during the 2004-05 influenza season. During this time, pediatricians prioritized vaccination of younger children and those with chronic medical conditions, such as chronic heart or kidney diseases and organ transplants or cancer. ${ }^{11}$ Another strategy used in emergency medicine during a past shortage of tetanus vaccine was to provide tetanus boosters to patients with high-risk wounds only. ${ }^{27}$

Although the tracking of current vaccine stocks and early notification of when these stocks fall below certain levels do not prevent vaccine shortages, these methods could initiate early mitigation measures even before a vaccine shortage hits by issuing preventive deferrals or modifying immunization recommendations. The 2002 vaccine shortage resulted in multiple at-risk children not being vaccinated according to schedule. ${ }^{20,22}$ In response, CDC used vaccine stockpiles for children to mitigate the risk of potential epidemics triggered by future shortages. One model suggested that while stockpiles were sufficient to meet future interruptions in manufacturing that last less than 6 months, the prolonged median shortages (5.1 months for immune globulins and 25.7 months for combination products) in this analysis (Table 1) suggest that such stockpiles may be inadequate to meet vaccination needs for children. ${ }^{22}$ A 6-month stockpile is therefore unlikely to be effective due to the typically lengthy duration of vaccine shortages.

While FDA has imported medications from other countries in the past to help alleviate drug shortages (e.g., propofol, nitroglycerin, doxorubicin), no vaccine products have been imported to help alleviate vaccine shortages, and doing so could lead to extra costs due to the additional regulatory approval process. The importation of vaccines would be required if a U.S. vaccine product was discontinued without the availability of an adequate alternative.
It would be useful to assess whether drug shortages in the United States also occur globally; however, the United States is one of the few countries with such a robust system for tracking drug shortages. Other countries do not have a systematic way of tracking drug shortages or their systems are in the course of development. Manufacturers of vaccines in multiple countries do not provide information about vaccines marketed outside the United States to clinicians in the United States. ${ }^{17}$ Despite this limitation, even if it is possible to know if a vaccine was marketed in another country, it is still impossible to know if there were shortages at that time in another country unless case reports were published in the literature, as it was for a shortage of bacille CalmetteGuérin vaccine in Europe. ${ }^{28}$

Ethical frameworks. Regardless of the type of vaccine implicated in a shortage, it is important to have an organized and ethical framework for the distribution of limited vaccine supplies. General ethical recommendations on the rationing of drugs respecting principles of justice and fairness include establishing an allocation committee for drugs on shortage to provide guidelines for patient prioritization. ${ }^{29}$ For vaccines represented in pediatric schedules, ACIP and CDC have often provided recommendations for prioritizing patients. A strategy used for transferring ethical principles into oncology practice regarding drug shortages is the so-called "A4R" approach, which addresses relevance, transparency, revision, and enforcement. ${ }^{30}$

A key difference between vaccine and immune globulin shortages and other drug shortages is that there is typically no immediate harm due to a shortage, but future harm may be long reaching if sufficient vaccines are deferred to reduce overall herd immunity. There are also fewer available alternatives for a vaccine shortage.

Addressing reasons for shortages. The vaccine manufacturing process is complex and thus fragile. A CDC 
publication described the vaccine shortages between 2000 and 2004 and the reason for the shortages in detail. ${ }^{19}$ Factors disturbing the manufacturing process include production problems, adherence to quality-control measures, recommendations to remove the preservative thimerosal, and economic decisions.

Production problems as a cause of shortages is not unique to vaccines. A summary of drug shortages (not limited to vaccines) found that injectable drugs accounted for $73 \%$ of all shortages in 2011 and that quality issues accounted for $56 \%$ of shortages of sterile injectable drugs. ${ }^{31}$ Quality issues for injectables were defined as bacterial or mold contamination and the presence of foreign particles in vials. These findings suggest that manufacturers should develop and expand their own quality-control measures in order to minimize these incidents. ${ }^{32} \mathrm{FDA}$, GAO, and the International Society for Pharmaceutical Engineering have identified quality issues and manufacturing problems as the key causes of drug shortages. It has been proposed that the reason for problems related to quality is a lack of transparency, which results in few manufacturers devoting resources to ensure the highest quality manufacturing. ${ }^{33}$

Ultimately, a multifaceted approach involving stakeholders from government and industry will be required to address the underlying reasons for vaccine shortages. Suggested solutions include financial incentives for vaccine manufacturers, regulatory changes, and rotating vaccine stockpiles, particularly for pediatric vaccines. ${ }^{20}$

Implications for pandemic preparedness. Influenza vaccine shortages have significant implications for preparedness for influenza epidemics and pandemics, particularly given the small number of influenza vaccine manufacturers. Even with 5 manufacturers of influenza vaccine, the total number of influenza vaccine doses provides a dose for less than half the population of the United States. ${ }^{34}$ In addition, the majority of influenza vaccine produced in the United States requires a lengthy egg-based manufacturing process. ${ }^{35}$ Cell-based and recombinant influenza vaccines are made by 2 separate companies but represent a low volume of the overall influenza vaccine market. ${ }^{34}$ Influenza vaccines were affected by 2 shortages during the observation period. While nonpharmaceutical interventions such as social distancing and postexposure prophylaxis will play a major role during a pandemic, vaccination remains an important strategy, particularly for at-risk populations. ${ }^{36}$ Alternative strategies during shortages include targeting and prioritizing high-risk groups for vaccination as well as using modified formulations (i.e., split or whole virus formulations or adjuvants). ${ }^{37}$

Additional preventive measures. CDC maintains a national stockpile that includes a 6-month rotating supply of pediatric vaccines, which began as early as 1983 for some vaccines. ${ }^{19}$ Since then, stockpiles have been used to cover vaccine demand on at least 8 occasions until 2004..$^{12}$ Optimization efforts concerning stockpiling pediatric vaccines were implemented in 2002. ${ }^{19}$

Several simulations exist to optimize vaccine stockpiling. ${ }^{38,39}$ Planned stockpiling at a national level must be differentiated from individual physicians' practices to maintain vaccine stocks in their offices, which is better described as hoarding. Furthermore, SWOT analysis (strengths, weaknesses, opportunities, threats) may help public health partners address shortages of vaccinations, such as influenza vaccine, to help develop strategies for rationing vaccines during public health emergencies. ${ }^{40}$

Study limitations. The major limitation of this study was that we could not assess the actual effect of shortages on individual institutions or patient care (e.g., delayed or suboptimal therapy, resultant medication errors and adverse outcomes). We also could not describe the severity of shortages affecting a particular product (i.e., whether the product was in limited supply or completely unavailable) and how these were ultimately dealt with on a healthcare system level. However, CDC adjusted the vaccination schedule for multiple products due to shortages of MMR vaccine, Hib vaccine, PCV7, and varicella vaccine. CDC also recommended deferring preexposure prophylaxis vaccinations during the rabies vaccine shortage. We did not take into account additional costs or toxicity when considering the use of available substitutes. In addition, the duration of shortages that were active at the end of the study period would be underestimated. Finally, we could not account for the time and resources used by institutions to mitigate drug shortages as well as the effectiveness of mitigation strategies. These are all potential areas for additional prospective studies.

\section{Conclusion}

Drug shortages of vaccines and immune globulin products accounted for only $2.8 \%$ of reported drug shortages within a 15-year period, but about half of these shortages involved products on the pediatric vaccination schedule, which may have significant public health implications.

\section{Disclosures}

The authors have declared no potential conflicts of interest.

\section{References}

1. Center for Drug Evaluation and Research. Drug shortage management. www.fda.gov/downloads/AboutFDA/ CentersOffices/OfficeofMedicalProductsandTobacco/CDER/ManualofPoliciesProcedures/UCM079936.pdf (accessed 2016 Nov 16).

2. U.S. Government Accountability Office. Drug shortages-public health threat continues, despite efforts to help ensure product availability. www.gao.gov/assets/670/660785.pdf (accessed 2016 Nov 16).

3. Decamp M, Joffe S, Fernandez CV et al. Chemotherapy drug shortages in pediatric oncology: a consensus statement. Pediatrics. 2014; 133:e71624. 
4. Hawley KL, Mazer-Amirshahi M, Zocchi MS et al. Longitudinal trends in U.S. drug shortages for medications used in emergency departments (2001-2014). Acad Emerg Med. 2016; 23:63-9.

5. Quadri F, Mazer-Amirshahi M, Fox ER et al. Antibacterial drug shortages from 2001 to 2013: implications for clinical practice. Clin Infect Dis. 2015; 60:1737-42.

6. Centers for Disease Control and Prevention. General recommendations on immunization: recommendations of the Advisory Committee on Immunization Practices (ACIP). MMWR. 2011; 60 (RR-2):1-64.

7. Briere EC, Jackson M, Shah SG et al. Haemophilus influenzae type b disease and vaccine booster dose deferral, United States, 1998-2009. Pediatrics. 2012; 130:414-20.

8. Thomas JD, Jackson ML, Sharma D et al. Haemophilus influenzae type b carriage among young children in metropolitan Atlanta in the context of vaccine shortage and booster dose deferral. Clin Vaccine Immunol. 2011; 18:2178-80.

9. White KE, Pabst LJ, Cullen KA. Upto-date Haemophilus influenzae type $b$ vaccination coverage during a vaccine shortage. Pediatrics. 2011; 127:e707-12.

10. Fairbrother G, Donauer S, Staat MA et al. Cincinnati pediatricians' measured and reported immunizing behavior for children during the national shortages of pneumococcal conjugate vaccine. Vaccine. 2013; 31:2177-83.

11. McQuillan L, Daley MF, Stokley S et al. Impact of the 2004-2005 influenza vaccine shortage on pediatric practice: a national survey. Pediatrics. 2009; 123:e186-92.

12. Fox ER, Birt A, James KB et al. ASHP guidelines on managing drug product shortages in hospitals and health systems. Am J Health-Syst Pharm. 2009; 66:1399-406.

13. U.S. Government Accountability Office. Drug shortages: FDA's ability to respond should be strengthened. www.gao.gov/assets/590/587000.pdf (accessed 2016 Dec 16).

14. Fox ER, Tyler LS. Managing drug shortages: seven years' experience at one health system. Am J Health-Syst Pharm. 2003; 60:245-53.

15. Centers for Disease Control and Prevention. National, state, and urban area vaccination cover- age among children aged 19-35 months-United States, 2003. www. cdc.gov/mmwr/preview/mmwrht$\mathrm{ml} / \mathrm{mm} 5329 \mathrm{a} 3 . \mathrm{htm}$ (accessed 2017 Mar 28).

16. Centers for Disease Control and Prevention. National, state, and urban area vaccination coverage among children aged 19-35 months-United States, 2005. www.cdc.gov/mmwr/ preview/mmwrhtml/mm5329a3.htm (accessed 2017 Mar 28).

17. Fox ER, Sweet BV, Jensen V. Drug shortages: a complex health care crisis. Mayo Clin Proc. 2014; 89:361-73.

18. U.S. Government Accountability Office. Drug shortages-certain factors are strongly associated with this persistent public health challenge. www.gao.gov/assets/680/678281.pdf (accessed 2017 Mar 27).

19. Rodewald LE, Orenstein WA, Mason DD et al. Vaccine supply problems: a perspective of the Centers for Disease Control and Prevention. Clin Infect Dis. 2006; 42(suppl 3):S104-10.

20. U.S. General Accounting Office. Childhood vaccines-ensuring an adequate supply poses continuing challenges. www.gao.gov/assets/240/235552.pdf (accessed 2016 Aug 16).

21. Groom H, Bhatt A, Washington ML et al. Temporary vaccine recommendations and provider compliance: a survey of pediatric practices during the 2003-2004 pneumococcal conjugate vaccine shortage. Pediatrics. 2008; 122:e835-40.

22. Jacobson SH, Sewell EC, Proano RA. An analysis of the pediatric vaccine supply shortage problem. Health Care Manag Sci. 2006; 9:371-89.

23. Groom AV, Cheek JE, Bryan RT. Effect of a national vaccine shortage on vaccine coverage for American Indian/ Alaska Native children. Am J Public Health. 2006; 96:697-701.

24. Iyengar S, Hedman L, Forte G et al. Medicine shortages: a commentary on causes and mitigation strategies. BMC Med. 2016; 14:124.

25. Mazer-Amirshahi M, Pourmand A, Singer $\mathrm{S}$ et al. Critical drug shortages: implications for emergency medicine. Acad Emerg Med. 2014; 21:704-11.

26. Broder KR, MacNeil A, Malone S et al. Who's calling the shots? Pediatricians' adherence to the 2001-2003 pneumococcal conjugate vaccine-shortage recommendations. Pediatrics. 2005; 115:1479-87.
27. Tyler LS, Fox ER, Caravati EM. The challenge of drug shortages for emergency medicine. Ann Emerg Med. 2002; 40:598-602.

28. Kontturi A, Santiago B, Tebruegge M et al. The impact of bacille CalmetteGuerin shortage on immunisation practice and policies in Europe-a Paediatric Tuberculosis Network European Trials Group (PTBNET) survey. Tuberculosis. 2016; 101:125-9.

29. Rosoff PM. Unpredictable drug shortages: an ethical framework for short-term rationing in hospitals. Am J Bioeth. 2012; 12:1-9.

30. Valgus J, Singer EA, Berry SR et al. Ethical challenges: managing oncology drug shortages. J Oncol Pract. 2013; 9:e21-3.

31. Kweder SL, Dill S. Drug shortages: the cycle of quantity and quality. Clin Pharmacol Ther. 2013; 93:245-51.

32. Fox ER, Tyler LS. Call to action: finding solutions for the drug shortage crisis in the United States. Clin Pharmacol Ther. 2013; 93:145-7.

33. Woodcock J, Wosinska M. Economic and technological drivers of generic sterile injectable drug shortages. Clin Pharmacol Ther. 2013; 93:170-6.

34. Centers for Disease Control and Prevention. Seasonal influenza vaccine supply for the U.S. 2016-2017 influenza season. www.cdc.gov/flu/ about/qa/vaxsupply.htm (accessed 2017 Apr 28).

35. Centers for Disease Control and Prevention. How influenza (flu) vaccines are made. www.cdc.gov/flu/protect/ vaccine/how-fluvaccine-made.htm (accessed 2017 Apr 28).

36. Jacobson SH, Sewell EC, Jokela JA. Survey of vaccine distribution and delivery issues in the USA: from pediatrics to pandemics. Expert Rev Vaccines. 2007; 6:981-90.

37. Hehme N, Engelmann H, Kunzel $\mathrm{W}$ et al. Pandemic preparedness: lessons learnt from H2N2 and H9N2 candidate vaccines. Med Microbiol Immunol. 2002; 191:203-8.

38. Shrestha SS, Wallace GS, Meltzer MI. Modeling the national pediatric vaccine stockpile: supply shortages, health impacts and cost consequences. Vaccine. 2010; 28:6318-32.

39. Truong VA. The pediatric vaccine stockpiling problem. Vaccine. 2012; 30:6175-9.

40. Uscher-Pines L, Barnett DJ, Sapsin JW et al. A systematic analysis of influenza vaccine shortage policies. Public Health. 2008; 122:183-91. 